\title{
Publisher Correction: Small targeted changes can yield substantial gains for human health and the environment
}

Katerina S. Stylianou (D), Victor L. Fulgoni III and Olivier Jolliet (D)

Correction to: Nature Food https://doi.org/10.1038/s43016-021-00343-4, published online 18 August 2021.

In the version of this article initially published, the title read "Small targeted dietary changes can yield substantial gains for human and environmental health." The title has now been corrected to read: "Small targeted changes can yield substantial gains for human health and the environment."

The title has been corrected in the online version of the paper.

Published online: 26 August 2021

https://doi.org/10.1038/s43016-021-00373-y

(c) The Author(s), under exclusive licence to Springer Nature Limited 2021 